\title{
THE STABILITY OF THE BERGMAN KERNEL AND THE GEOMETRY OF THE BERGMAN METRIC
}

\author{
BY ROBERT E. GREENE ${ }^{1}$ AND STEVEN G. KRANTZ ${ }^{2}$
}

If $D$ is a bounded open subset of $\mathbf{C}^{n}$, the set $H=\{f: D \rightarrow \mathbf{C} \mid f$ is holomorphic and $\left.\int_{D}|f|^{2}<+\infty\right\}$ is a separable infinite-dimensional Hilbert space relative to the inner product $\langle f, g\rangle=\int_{D} f \bar{g}$. The completeness of $H$ can be seen from Cauchy integral estimates. Similar estimates show that for any $p \in D$ the functional $f \mapsto f(p), f \in H$, is continuous. Thus there is a unique element $K_{D}(z, p) \in H$ (as a function of $z$ ) such that,

$$
f(p)=\int f(z) \overline{K_{D}(z, p)} d V(z) \text { for all } f \in H .
$$

The function $K_{D}$ is called the Bergman kernel function. If $\left\{\varphi_{i}\right\}_{i=1}^{\infty}$ is an orthonormal basis for $H$ then $K_{D}(z, p)=\Sigma_{i} \varphi_{i}(z) \overline{\varphi_{i}(p)}$. The convergence of the series is absolute, uniformly on compact subsets of $D \times D$. For any $z \in D, K_{D}(z, z)>0$ and $\log K_{D}(z, z)$ is a real analytic function on $D$. The Hermitian form

$$
\sum_{i, j} \frac{\partial^{2}}{\partial z_{i} \partial \bar{z}_{j}} \log K_{D}(z, z) d z_{i} \otimes d \bar{z}_{j}
$$

is positive definite on $D$ and defines a Kähler metric on $D$ called the Bergman metric of $D$. Calculations using orthonormal bases show that if $F: D_{1} \rightarrow D_{2}$ is a biholomorphic mapping then $F$ is an isometry of the Bergman metric of $D_{1}$ to that of $D_{2}$. Thus differential geometric methods can be used to study such mappings. The Bergman metric is usually hard to compute explicitly; but, especially in the case of $C^{\infty}$ strongly pseudoconvex domains, considerable information can be obtained by indirect methods. The starting point for such investigation is the observation that $K_{D}(\cdot, p)$ is, in a suitable sense, the orthogonal projection on $H$ of the delta function $\delta_{p}$. This projection can be expressed in terms of the solution of the $\bar{\partial}$-Neumann problem; a set of powerful techniques is thereby brought to bear on the matter. With these techniques, extensive information about the behavior of $K_{D}(z, p)$ for $p, z$ near the boundary of $D$ has been obtained [9] , [3].

Received by the editors July 11,1980 .

1980 Mathematics Subject Classification. Primary 32H10, 35N15; Secondary 32G05, $32 \mathrm{H} 05,53 \mathrm{C} 55$.

${ }^{1}$ Research supported in part by NSF Grant \#MCS79-01062, The Institute for Advanced Study, and an Alfred P. Sloan Foundation Fellowship.

${ }^{2}$ Research supported by the National Science Foundation MCS77-02213. 
This announcement presents a number of results about the stability properties of the Bergman kernel and metric under perturbations of the boundary of $C^{\infty}$ strongly pseudoconvex domains and some applications of these stability properties to the geometry of the Bergman metric. Further results, proofs in detail, etc. are given in the authors' papers [5]-[7].

1. The stability of the Bergman kernel. If $\left\{K_{i}\right\}_{i=1}^{\infty}$ is a sequence of compact subsets of $\mathbf{R}^{k}$ then, by definition, $\left\{K_{i}\right\}_{i=1}^{\infty}$ converges $C^{\infty}$ to a compact set $K \subseteq \mathbf{R}^{k}$ if there is a sequence $h_{i}: K \rightarrow \mathbf{R}^{k}$ of $C^{\infty}$ diffeomorphisms such that $h_{i}(K)=K_{i}$ and $h_{i}$ converges to the identity in the $C^{\infty}$ topology of maps of compact subsets of $\mathbf{R}^{k}$. (Here $h: K \rightarrow \mathbf{R}^{k}$ is called a diffeomorphism if it is the restriction to $K$ of a diffeomorphism into $\mathbf{R}^{k}$ of some open set containing $K$.) If $K_{i} \rightarrow K$ in the $C^{\infty}$ topology and $f_{i} \in C^{\infty}\left(K_{i}\right)$ then $f_{i} \rightarrow f \in C^{\infty}(K)$ in $C^{\infty}$ if $f_{i} \circ h_{i} \rightarrow f$ in $C^{\infty}$ in the usual sense. These convergence ideas define topologies hereinafter called the $C^{\infty}$ topology.

For any bounded domain $D \subseteq \mathbf{C}^{n}$, let $E_{D}^{\delta}=\{(z, w) \in \bar{D} \times \bar{D}: \operatorname{dis}(z, w)+$ $\operatorname{dis}(z, \partial D)+\operatorname{dis}(w, \partial D)<\delta\}$. If $D$ is a $C^{\infty}$ strongly pseudoconvex domain then, for any $\delta>0$, the Bergman kernel $K_{D}$ extends to be a $C^{\infty}$ function on $\bar{D} \times \bar{D} \backslash E_{\delta}[9]$.

THEOREM 1. If $\left\{D_{i}\right\}_{i=1}^{\infty}$ is a sequence of $C^{\infty}$ strongly pseudoconvex domains converging in $C^{\infty}$ to a $C^{\infty}$ strongly pseudoconvex domain $D_{0}$ then, for each $\delta>0, K_{D_{i}}: \bar{D}_{i} \times \bar{D}_{i} \backslash E_{D_{i}}^{\delta} \rightarrow \mathbf{C}$ converges in $C^{\infty}$ to $K_{D_{0}}: \bar{D}_{0} \times \bar{D}_{0} \backslash E_{D_{0}}^{\delta} \rightarrow \mathbf{C}$.

If $D$ is a $C^{\infty}$ strongly pseudoconvex domain then, for sufficiently small $\delta>0, K_{D}$ has a specific asymptotic expansion on $E_{D}^{\delta}$ ([3] ; also [1]). To formulate it, let $\psi$ be a $C^{\infty}$ strictly plurisuperharmonic defining function for $D(\psi>0$ on $D,<0$ on $\mathbf{C}^{n} \backslash \bar{D}, \operatorname{grad} \psi \neq 0$ on $\partial D$ ) and let

$$
\begin{aligned}
X_{\psi}(z, w)= & \psi(w)+\sum_{i}\left(\partial \psi /\left.\partial w_{i}\right|_{w}\right)\left(z_{i}-w_{i}\right) \\
& +\frac{1}{2} \sum_{i, j}\left(\partial^{2} \psi /\left.\partial w_{i} \partial w_{j}\right|_{w}\right)\left(z_{i}-w_{i}\right)\left(z_{j}-w_{j}\right) .
\end{aligned}
$$

If $\delta$ is sufficiently small, then there are $C^{\infty}$ functions $\varphi(z, w)$ and $\widetilde{\varphi}(z, w)$ on $\bar{E}_{D}^{\delta}$ such that, on $(D \times D) \cap E_{D}^{\delta}, K_{D}(z, w)=\varphi(z, w) X_{\psi}^{-(n+1)}(z, w)+$ $\widetilde{\varphi}(z, w) \log X_{\psi}(z, w)$.

THEOREM 2. If $\left\{D_{i}\right\}_{i=1}^{\infty}$ is a sequence of $C^{\infty}$ strongly pseudoconvex domains converging in $C^{\infty}$ to a $C^{\infty}$ strongly pseudoconvex domain $D_{0}$ then there exist $a \delta>0$ and $C^{\infty}$ functions $\psi_{i}: \mathbf{C}^{n} \rightarrow \mathbf{R}, \varphi_{i}: \bar{E}_{D_{i}}^{\delta} \rightarrow \mathbf{C}, \widetilde{\varphi}_{i}: \bar{E}_{D_{i}}^{\delta} \rightarrow \mathbf{C}$, $i=0,1,2, \ldots$, such that $\left\{\psi_{i} \mid D_{i}\right\}$ converges $C^{\infty}$ to $\psi_{0},\left\{\varphi_{i}\right\}$ converges $C^{\infty}$ 
to $\varphi_{0},\left\{\widetilde{\varphi}_{i}\right\}$ converges $C^{\infty}$ to $\widetilde{\varphi}_{0}$ and such that for all $i=0,1,2, \ldots$ it holds that $K_{D_{i}}(z, w)=\varphi_{i}(z, w) X_{\psi}^{-(n+1)}(z, w)+\tilde{\varphi}_{i}(z, w) \log X_{\psi_{i}}(z, w)$.

The proofs of Theorems 1 and 2 involve, among other ingredients, the formulation and proof of certain stability results for the Kohn solution of the $\bar{\partial}$ equation (certain specific instances of this kind of stability have been indicated in [4]).

2. Stability of the geometry of the Bergman metric. The Bergman metric of a $C^{\infty}$ strongly pseudoconvex domain is complete so that in the presence of curvature information the methods of global Riemannian geometry can be applied. The stability of curvature away from the boundary follows immediately from Theorem 1. In fact, curvature behavior is globally stable since it is also stable near the boundary.

THEOREM 3. If $D_{0}$ is a $C^{\infty}$ strongly pseudoconvex domain then, for any $\epsilon>0$, there is a $C^{\infty}$ neighborhood $U$ of $D_{0}$ and a $\delta>0$ such that the following holds. For any $D \in U$ and any holomorphic 2-plane $P$ at any point $p \in D$ with $\operatorname{dis}(p, \partial D)<\delta$ it is the case that $|\kappa(P)+4 /(n+1)|<\epsilon$, where $\kappa$ is the sectional curvature of the Bergman metric of $D$.

The existence of $\delta$ for a single domain is shown in [10] (see also [2]). A stability result also holds for covariant derivatives of the curvature. These stabilities follow by calculations (cf. [10]) using the stability of the expansion of the Bergman kernel (Theorem 2).

The Bergman metric of a domain in $\mathbf{C}^{n}$ has constant holomorphic sectional curvature (if and) only if the domain is biholomorphic to the ball [11]. This fact, Theorem 3, and normal families arguments imply

(i) the set $\left\{D: D\right.$ is $C^{\infty}$, strictly pseudoconvex, and $\left.\operatorname{Aut}(D)=\{\mathrm{id}\}\right\}$ is $C^{\infty}$ open, and

(ii) the set $\left\{\left(D_{1}, D_{2}\right): D_{1}, D_{2}\right.$ are $C^{\infty}$, strictly pseudoconvex, and $D_{1}$ is biholomorphic to $\left.D_{2}\right\}$ is closed in the product $C^{\infty}$ topology on $\left\{C^{\infty}\right.$ strongly pseudoconvex domains $\} \times\left\{C^{\infty}\right.$ strongly pseudoconvex domains $\}$.

Here $\operatorname{Aut}(D)$ denotes the group of biholomorphic self maps of $D$. Note that (i) and (ii) do not follow from Chern-Moser invariant theory since the obstructions to the existence of automorphisms or equivalences may be global in nature and not detectable by local invariants. Fact (i) is a special case of a result on semicontinuity of automorphism groups (see [7]).

3. Perturbations of the ball. It is a special case of the curvature results of $\S 2$ that there is a $C^{\infty}$ neighborhood $U$ of the unit ball in $\mathbf{C}^{n}$ such that, for every $D \in U$, the Bergman metric of $D$ has everywhere negative Riemannian sectional curvature. In fact for suitable $U$ the curvature of $D \in U$ will be globally uniformly close to the curvature of the ball. If $D \in U$ but $D$ is not biholomorphic 
to the ball, then $\operatorname{Aut}(D)$ is necessarily compact [14]. Moreover, $\operatorname{Aut}(D)$ then has a fixed point since the Bergman metric has nonpositive curvature and a compact group of isometries of a complete simply connected manifold of nonpositive curvature has a fixed point (E. Cartan's Theorem).

Theorem 4. For each $\epsilon>0$ there is a $C^{\infty}$ neighborhood $U$ of the ball $B \subseteq \mathrm{C}^{n}$ such that if $D \in U$, if $D$ is not biholomorphic to $B$, and if the (necessarily nonempty) fixed point set of $\operatorname{Aut}(D)$ contains a point $p \in D$ with $\operatorname{dis}(p, \partial D)>\epsilon$ then there is a $C^{\infty}$ bounded domain $D^{\prime}$ in $\mathbf{C}^{n}$ such that $D^{\prime}$ is biholomorphic to $D$ and such that the action of $\operatorname{Aut}\left(D^{\prime}\right)$ on $D^{\prime}$ is the restriction to $D^{\prime}$ of the linear action on $\mathbf{C}^{n}$ of a subgroup of the unitary group.

Theorem 4 follows from Theorem 1 via Bergman representative coordinates.

The curvature stability results of $\S 2$ are in a sense the best possible for perturbations of the ball.

THEOREM 5. If $M$ is a simply connected complex manifold and if for every $\epsilon>0$ there is on $M$ a complete Kähler metric with all holomorphic sectional curvature between $-1-\epsilon$ and -1 , then $M$ is biholomorphic to the unit ball in $\mathbf{C}^{n}, n=\operatorname{dim}_{\mathbf{C}} M$.

From Theorem 5, the rigidity results of [13], and the finiteness theorem of [8], it follows that for each $n \geqslant 1$ and $c>0$ there is an $\epsilon>0$ such that any compact Kähler manifold $M$ with $\operatorname{dim}_{\mathrm{C}} M=n$, volume $M<c$, and holomorphic sectional curvature between $-1-\epsilon$ and -1 is holomorphically covered by the ball in $\mathbf{C}^{n}$ (also proved by M. Gromov by a different method; the authors are indebted to E. Ruh for pointing out the relevance of Theorem 5 to finiteness results for compact manifolds).

Sufficiently small $C^{\infty}$ neighborhoods $U$ of $B$ provide an infinite-dimensional family of biholomorphically inequivalent complete Kähler manifolds of strictly negative Riemannian sectional curvature. Details appear in [5]. Previously, the only known example (which is not known to be a domain) not biholomorphic to the ball was that in [12].

\section{REFERENCES}

1. L. Boutet de Monvel and J. Sjöstrand, Sur la singularité des noyaux de Bergman et de Szegö, Soc. Mat. de France Asterisque 34-35 (1976), 123-164.

2. S.-Y Cheng and S. T. Yau, On the existence of complete Kähler metrics on noncompact manifolds and the regularity of Fefferman's equation, Comm. Pure Appl. Math. 23 (1980), 507-544.

3. C. Fefferman, The Bergman kernel and biholomorphic mappings of pseudoconvex domains, Invent. Math. 26 (1974), 1-65.

4. G. B. Folland and J. J. Kohn, The Neumann problem for the Cauchy-Riemann complex, Princeton Univ. Press, Princeton, N. J., 1972. 
5. R. E. Greene and S. G. Krantz, Stability of the Bergman kernel and curvature properties of bounded domains, Proc. Princeton Conf. on Several Complex Variables, 1979 (to appear).

6. Deformation of complex structures, estimates for the $\bar{\partial}$ equation, and stability of the Bergman kernel, Advances in Math. (to appear).

7. - The automorphism groups of strongly pseudoconvex domains (to appear).

8. M. Gromov, Manifolds of negative curvature, J. Differential Geometry 13 (1978), 223-230.

9. N. Kerzman, The Bergman kernel function. Differentiability at the boundary, Math. Ann. 195 (1972), 149-158.

10. P. Klembeck, Kähler metrics of negative curvature, the Bergman metric near the boundary and the Kobayashi metric on smoothly bounded strictly pseudoconvex sets, Indiana Univ. Math. J. 27 (1978), 275-282.

11. Lu Qi-Keng (= K. H. Look), On Kähler manifolds with constant negative curvature, Acta Math. Sinica 16 (1966), 269-281 (Chinese) = Chinese Math. 9 (1966), 283-298.

12. G. D. Mostow and Y. T. Siu, A compact Kähler surface of negative curvature not covered by the ball, Ann. of Math. 112 (1980), 321-360.

13. Y. T. Siu, The complex analyticity of harmonic maps and the strong rigidity of compact Kähler manifolds, Ann. of Math. 112 (1980), 73-112.

14. B. Wong, Characterizations of the ball in $\mathrm{C}^{n}$ by its automorphism group, Invent. Math. 41 (1977), 253-257.

DEPARTMENT OF MATHEMATICS, UNIVERSITY OF LOS ANGELES, LOS ANGELES, CALIFORNIA 90024

Current address: (Steven G. Krantz) DEPARTMENT OF MATHEMATICS, PRINCETON UNIVERSITY, PRINCETON, NEW JERSEY 08540 